\title{
HERPETOFAUNA DEL CERRO GUIENGOLA, ISTMO DE TEHUANTEPEC, OAXACA
}

\author{
Cintia Natalia Martín-Regalado, ${ }^{1,2}$ Rosa Ma. Gómez-Ugalde ${ }^{1}$ \\ y Ma. Emma Cisneros-Palacios ${ }^{2}$ \\ ${ }^{1}$ Instituto Tecnológico del Valle de Oaxaca, Ex Hacienda de Nazareno, Xoxocotlán, Oaxaca. \\ C. P. 68000.<cinthia_14@msn.com.mx> \\ ${ }^{2}$ CIIDIR-IPN. Unidad Oaxaca. Calle Hornos 1003 Xoxocotlán, Oaxaca.
}
Cintia Natalia Martín-Regalado, C. N., R. M. Gómez-Ugalde \& M. E. Cisneros-Palacios. 2011. Herpetofauna del Cerro Guiengola, Istmo de Tehuantepec, Oaxaca. Acta Zoológica Mexicana (n. s.), 27(2): 359-376.

RESUMEN. Este estudio se realizó en el Cerro Guiengola, Tehuantepec, Oaxaca, con el objetivo de contribuir al conocimiento de la herpetofauna en dicha área. Se obtuvieron 602 registros visuales y se recolectaron 103 ejemplares de anfibios y reptiles durante 60 días de trabajo de campo. Se enlistan 40 especies, pertenecientes a 33 géneros y 18 familias. Se determinó la distribución de la herpetofauna en el Cerro Guiengola por microhábitat, tipo de vegetación y altitud. Usando el método de Jaccard se elaboraron dendrogramas de similitud por microhábitat y tipo de vegetación. Se reportan especies por tipo de vegetación y rangos altitudinales diferentes a los citados en la literatura. De acuerdo con los valores obtenidos, los índices de diversidad de Simpson y Shannon-Wiener demuestran que el área de estudio es diversa. De las 40 especies que se registraron, 13 son endémicas de México y tres de Oaxaca. El 42.5\% de la herpetofauna registrada se encuentra enlistada en la NOM-059-ECOL-2001 y el 47.5\% en la Unión Internacional para la Conservación de la Naturaleza (IUCN).

Palabras clave: Herpetofauna, Cerro Guiengola, Istmo de Tehuantepec, microhábitat, vegetación, distribución altitudinal, diversidad, especies en riesgo.

Cintia Natalia Martín-Regalado, C. N., R. M. Gómez-Ugalde \& M. E. Cisneros-Palacios. 2011. Herpetofauna of the Cerro Guiengola, Isthmus of Tehuantepec, Oaxaca. Acta Zoológica Mexicana (n. s.), 27(2): 359-376.

ABSTRACT. This study was performed in Cerro Guiengola in the Mexican state of Oaxaca, located in the Isthmus of Tehuantepec, with the objective of contributing to the knowledge on herpetofauna located in that area; 103 collections and 602 visual records of amphibians and reptiles were made during 60 days of field work, and 40 species belonging to 32 genera and 18 families are listed in this study. Based on the data collected, the herpetofauna distribution in Cerro Guiengola was classified according to altitude, vegetation and microhabitat. Vegetation and microhabitat similarity dendograms were made

Recibido: 11/06/2010; aceptado: 18/02/2011. 
using Jaccard's method. Some species were reported in altitudes and vegetation types different from those cited in the literature. Simpson and Shannon-Wiener's indices of diversity show that the study area varies according to the obtained data; 13 species out of the 40 species recorded are endemic to Mexico and 3 to Oaxaca; $42.5 \%$ of the reported herpetofauna are listed in the NOM-059-ECOL-2001 and 47.5\% by the International Union for the Conservation of Nature (IUCN).

Key words: Herpetofauna, Cerro Guiengola, Isthmus of Tehuantepec, microhabitat, vegetation, altitudinal distribution, diversity, threatened species.

\section{INTRODUCCIÓN}

El estado de Oaxaca se encuentra ubicado al sur de la República Mexicana, en la parte más estrecha del país, tiene vertientes hacia el Golfo de México y el Océano Pacífico así como un sistema orográfico complejo formado por la Sierra Madre del Sur, la Sierra Madre de Oaxaca, continuación de la Sierra Madre Oriental, y la Sierra Atravesada (López 1979). Presenta variados tipos de climas que van desde los cálidos a húmedos-áridos, pasando por los semicálidos, hasta los semifríos influenciados por los ciclones o huracanes (Martínez-Ramírez 1999). Todo lo anterior propicia la existencia de diferentes tipos de comunidades vegetales que aunado a su historia biogeográfica compleja, favorecen la existencia de una de las faunas terrestres más diversas de México, con presencia de una gran cantidad de endemismos. Esta fauna representa un complejo de formas neotropicales y neárticas que alcanzan en el territorio oaxaqueño el límite de su distribución (Canseco 1996; Casas-Andreu et al. 1996, 2004), haciendo de Oaxaca el estado mexicano más rico en anfibios y reptiles (Hartweg \& Oliver 1937a, 1937b, 1938, 1940; Smith \& Taylor 1966; Flores-Villela 1993; Flores \& Gerez 1994; Casas-Andreu et al. 1996, 2004).

El presente trabajo tuvo como objetivo estudiar la herpetofauna del Cerro Guiengola y determinar la distribución de las especies en los diferentes tipos de vegetación, microhábitats y cotas altitudinales.

\section{MATERIAL Y MÉTODOS}

Área de estudio. El Cerro Guiengola se localiza a $14 \mathrm{~km}$ al noroeste de la Ciudad de Santo Domingo Tehuantepec, entre los paralelos $16^{\circ} 21^{\prime}$ y $16^{\circ} 26^{\prime}$ de latitud norte y $95^{\circ} 17^{\prime}$ y $95^{\circ} 24^{\prime}$ de longitud oeste, ocupa una superficie aproximada de 4530 ha y su altura máxima es de $1050 \mathrm{~m}$ snm (Fig. 1). Está limitado al noroeste por la presa Benito Juárez, al sureste por la Ciudad de Santo Domingo Tehuantepec, al oeste por el Cerro El Portillo del Guayabo y al este por el poblado de Santa María Mixtequilla (Torres-Colín 1989).

La vegetación predominante en el área de estudio corresponde a las selvas bajas caducifolia y caducifolia espinosa, las especies arbóreas más abundantes son: Ceiba parvifolia, Fouqueria formosa, Bursera simaruba, Plumeria rubra y Lysiloma di- 


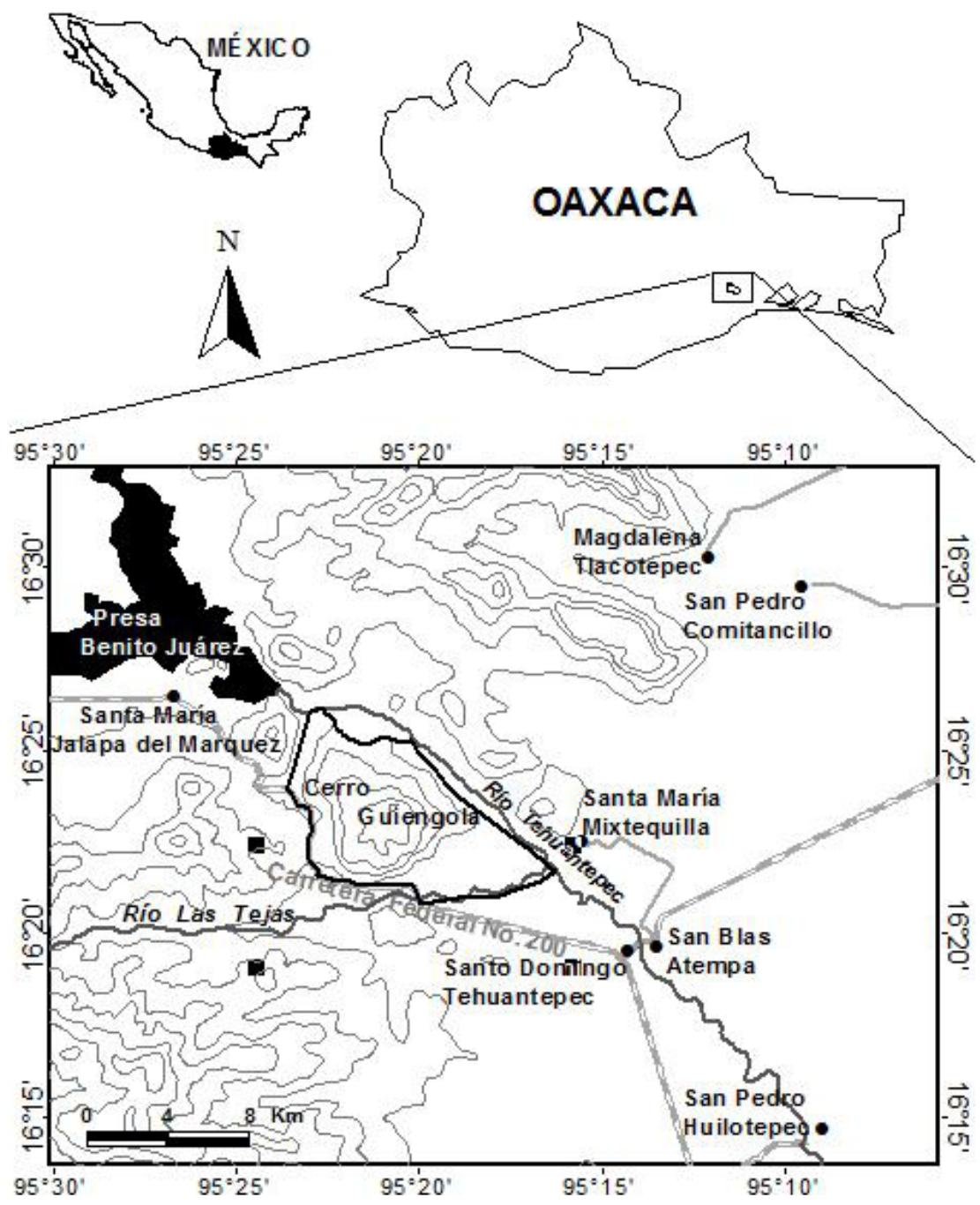

Figura 1. Localización del Cerro Guiengola, Tehuantepec, Oaxaca.

varicata; además de los árboles, las formas básicas de estas selvas son los arbustos, lianas y hierbas, también se hallan cactáceas y plantas de formas arrosetadas que se encuentran representadas por especies de los géneros Agave, Cephalocereus, Escontria, Pereskiopsis y Stenocereus (Torres-Colín 2004). Además de las selvas secas en el Cerro Guiengola, se encuentra un bosque de pino (Pinus michoacana), que se presenta como un manchón de vegetación de poca extensión en la parte más alta del cerro de 1000 a $1050 \mathrm{~m} \mathrm{snm}$. Al sureste y este del área de estudio se encuentra 
vegetación riparia con composición florística variable, las especies comunes son $\mathrm{Pi}$ thecellobium dulce, Lantana camara y Cnidoscolus spp. (Fig. 2). El origen de la roca madre es sedimentario y los suelos son someros y generalmente pedregosos (TorresColín 2004). De acuerdo con el sistema de clasificación climática de Köppen modificado por García (1988), los tipos de climas presentes en el área de estudio son: Awo (cálido subhúmedo) con lluvias en verano de 5 al 10.2\% anual; y BS1(h')w (cálido semiárido) con lluvias en verano de 5 al $10 \%$ anual.

Trabajo de campo. Se realizaron muestreos mensuales con una duración de cinco días cada uno, durante un año, de julio de 2007 a junio de 2008. En cada sesión se aplicó un esfuerzo de muestreo de dos a tres personas por día; se hicieron recorridos al azar y colectas diurnas, crepusculares y nocturnas para cubrir las diferentes horas de actividad de los anfibios y reptiles. En los recorridos se revisaron los microhábitats en los que potencialmente se podrían localizar estos organismos y se registró el tipo de vegetación en el que se encontraron. Se clasificaron los microhábitats, de acuerdo a su uso, utilizando las categorías terrestre, arborícola, ripario, saxícola y paredes y grietas en habitaciones humanas (Gutiérrez-Mayén \& Salazar 2006). Los ejemplares de tortugas y algunos anfibios y lagartijas se capturaron con la ayuda de ligas de hule, resorteras y con la mano; las serpientes se capturaron con ayuda de una pinza, un gancho herpetológico y guantes de carnaza. Para cada ejemplar capturado se registró: localidad, tipo de vegetación, fecha, hora de captura, altitud, microhábitat y obser-

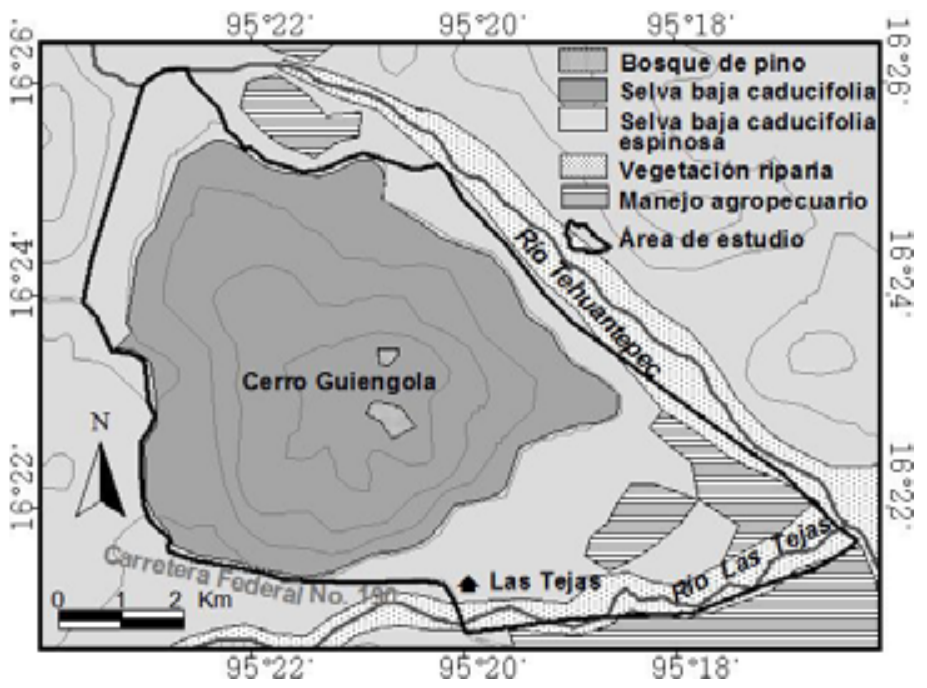

Figura 2. Tipos de vegetación en la zona de estudio. Fuente: Torres-Colín (1989); CONABIO (1999, 2006). 
vaciones sobre sexo, talla, coloración, etc. Para trasladar a los reptiles se utilizaron bolsas de manta y para los anfibios botes de plástico; posteriormente se sacrificaron los organismos bajándoles la temperatura corporal. La determinación taxonómica se llevó a cabo con las claves de identificación de anfibios y reptiles de Smith \& Taylor (1945, 1948, 1950), Casas-Andreu \& McCoy (1979), Flores-Villela et al. (1995), para la actualización nomenclatural se utilizó lo propuesto por Flores-Villela \& Canseco-Márquez (2004), Frost et al. (2006), Liner (2007) y Oliver-López et al. (2009). Antes de preparar los ejemplares, a cada individuo se les registró el largo total, largo cabeza-cloaca y el peso húmedo, además se anotó su coloración. Los ejemplares se fijaron con formol al 10\% y se conservaron en alcohol al 70\%. Las muestras curatoriales de los ejemplares fueron ingresadas en las Colecciones Batracológica y Herpetológica del CIIDIR-IPN-Oaxaca.

Análisis de datos. Para la clasificación de las especies se siguió el ordenamiento de Casas-Andreu et al. (2004) incluyendo los cambios taxonómicos de Flores-Villela \& Canseco-Márquez (2004) y Frost et al. (2006). Los registros obtenidos se sistematizaron por microhábitat, tipo de vegetación y distribución altitudinal obteniéndose la riqueza específica de estos. Para el análisis altitudinal se establecieron arbitrariamente siete pisos altitudinales en el Cerro Guiengola, en intervalos de $150 \mathrm{~m}$. Para el análisis por tipo de vegetación se utilizó la clasificación asignada por Torres-Colín (1989) para el Cerro Guiengola equivalente al utilizado por Flores-Villela \& Gerez (1994).

Con el fin de determinar si el esfuerzo de muestreo fue suficiente para registrar la riqueza herpetofaunística presente, se utilizaron los modelos de Dependencia Lineal y Clench para obtener las curvas de acumulación de especies con el programa Species Accumulation Functions Versión Beta (CIMAT 2003), el primero se utiliza para taxa bien conocidos o áreas relativamente pequeñas en que teóricamente se puede alcanzar la asíntota en períodos de tiempo finitos (Jiménez-Valverde \& Hortal 2000), mientras que el segundo está recomendado para estudios en sitios grandes y para protocolos en los que la probabilidad de añadir nuevas especies al inventario es mayor cuanto más tiempo se pasa en el campo (Soberón \& Llorente 1993). Con el objeto de eliminar el efecto del orden en que se ingresan los datos (Moreno \& Halffter 2000), se aleatorizaron previamente 100 veces los datos con ayuda del programa EstimateS Versión 8 (Colwell 2009). Debido a que no se alcanzó la asíntota, se calculó el esfuerzo de colecta adicional requerido para tener representado el $95 \%$ de las especies de la zona de trabajo.

Diversidad y abundancia. Se estimaron la diversidad alfa (riqueza de especies de una comunidad particular a la que se considera homogénea) y beta (grado de cambio o reemplazo en la composición de especies entre diferentes comunidades en un paisaje) en el área de estudio. La diversidad alfa se estimó mediante el cálculo del índice de Chao2 (recomendable para muestras pequeñas), el índice de dominancia de Simpson, que considera la probabilidad de que dos individuos tomados de una muestra sean 
de la misma especie y el índice de equidad de Shannon-Wiener, que asume que los individuos provienen de una comunidad de tamaño finito y que todas las especies están representadas (Whittaker 1972; Magurran 1988; Moreno 2001); estos índices consideran la distribución proporcional de cada especie (abundancia relativa de los individuos). Tanto el índice de Simpson como el de Shannon-Wiener se calcularon por temporada del año y para toda la zona de estudio.

Para estimar la diversidad beta se comparó la similitud de la riqueza de especies entre los tipos de vegetación y microhábitats con el coeficiente de Jaccard, con base en la presencia y ausencia de especies, los dendrogramas de similitud se elaboraron con el programa estadístico BioDiversity Professional Beta Versión 2 (McAleence 1997).

Especies en riesgo. Se revisaron los listados de especies de la NOM-059-ECOL2001 (SEMARNAT 2002), la IUCN (2009) y los apéndices de la CITES (2009) para determinar las especies de la zona de estudio bajo alguna categoría de riesgo.

\section{RESULTADOS}

Composición de la herpetofauna. En 12 muestreos con un total de 60 días efectivos de trabajo de campo se obtuvieron 602 registros visuales y se recolectaron 103 ejemplares, correspondientes a 40 especies, 33 géneros y 18 familias de anfibios y reptiles (Cuadro 1).

Las familias mejor representadas fueron Colubridae con 10 especies y Phrynosomatidae con seis. Las especies con mayor abundancia absoluta (número de ejemplares capturados y registrados) fueron Sceloporus siniferus siniferus, S. variabilis smithi, Urosaurus bicarinatus anonymorphus, Aspidoscelis deppii deppii y A. guttata immutabilis (Fig. 3).

Riqueza de especies por microhábitat. En relación con los tipos de microhábitats en que se registraron los reptiles en el Cerro Guiengola, se encontraron 27 especies en el terrestre, seguido por el ripario con 20; el de menor riqueza fue el de paredes y grietas en habitaciones humanas (Cuadro 2). Los anfibios usan menos ambientes debido a sus hábitos de vida, encontrándose básicamente en zonas riparias, aunque Ollotis canalifera y Rhinella marina frecuentemente se localizaron también en el ambiente terrestre.

Riqueza de especies por tipo de vegetación. La riqueza herpetofaunística en el Cerro Guiengola es mayor en las selvas bajas caducifolia y caducifolia espinosa, con 24 y 26 especies respectivamente (Cuadro 2), en el bosque de pino solo se registró una especie (Mabuya unimarginata).

Las especies más comunes en la selva baja caducifolia, selva baja caducifolia espinosa y vegetación riparia fueron Aspidoscelis d. deppii, Sceloporus variabilis smithi, S. siniferus siniferus y Urosaurus bicarinatus anonymorphus. 
Cuadro 1. Composición de la herpetofauna en el Cerro Guiengola, Tehuantepec, Oaxaca.

\begin{tabular}{|c|c|c|c|c|c|}
\hline Clase & Orden & Suborden & Familias & Géneros & Especies \\
\hline \multirow[t]{3}{*}{ Amphibia } & Anura & & Bufonidae & 2 & 2 \\
\hline & & & Hylidae & 1 & 1 \\
\hline & & & Leptodactylidae & 1 & 1 \\
\hline \multirow[t]{15}{*}{ Reptilia } & Squamata & Sauria & Corythophanidae & 1 & 1 \\
\hline & & & Gekkonidae & 3 & 3 \\
\hline & & & Iguanidae & 2 & 3 \\
\hline & & & Phrynosomatidae & 3 & 6 \\
\hline & & & Polychridae & 1 & 1 \\
\hline & & & Scincidae & 1 & 1 \\
\hline & & & Teiidae & 1 & 3 \\
\hline & & & Xantusiidae & 1 & 1 \\
\hline & & Serpentes & Boidae & 1 & 1 \\
\hline & & & Colubridae & 9 & 10 \\
\hline & & & Elapidae & 1 & 1 \\
\hline & & & Leptotyphlopidae & 1 & 1 \\
\hline & & & Loxocemidae & 1 & 1 \\
\hline & & & Viperidae & 2 & 2 \\
\hline & Testudines & & Emydidae & 1 & 1 \\
\hline Total & & & 18 & 33 & 40 \\
\hline
\end{tabular}

Algunas de las especies presentes en el Cerro Guiengola utilizan tipos de vegetación diferentes a los citados en la literatura (Flores \& Gerez 1994), ejemplos de estas especies son: Anolis compressicaudus, Ctenosaura oaxacana, Drymarchon melanurus, Masticophis mentovarius, Oxybelis aeneus, Rhinoclemmys rubida rubida, Sphaerodactylus glaucus, Symphimus leucostomus y Tantilla rubra; en total son 59 registros novedosos (Cuadro 2).

Distribución altitudinal de la herpetofauna del Cerro Guiengola. Dos especies se registraron en los siete pisos altitudinales, A. compressicaudus y Sceloporus s. siniferus, mostrando así que son las especies que ocupan un mayor intervalo altitudinal en el Cerro Guiengola; 15 especies, entre ellas Trimorphodon biscutatus, Drymarchon melanurus, Basiliscus vittatus, Phrynosoma asio, Ollotis canalifera y Rhinella marina solo se registraron en el primer piso altitudinal por debajo de los $150 \mathrm{~m} \mathrm{snm}$.

Varias especies fueron encontradas a altitudes diferentes que las reportadas por Casas-Andreu et al. (1996), observándose que Ctenosaura oaxacana (55-605 m snm), Sceloporus edwardtaylori (43-64 m snm) y Porthidium dunni (91-650 m snm) 


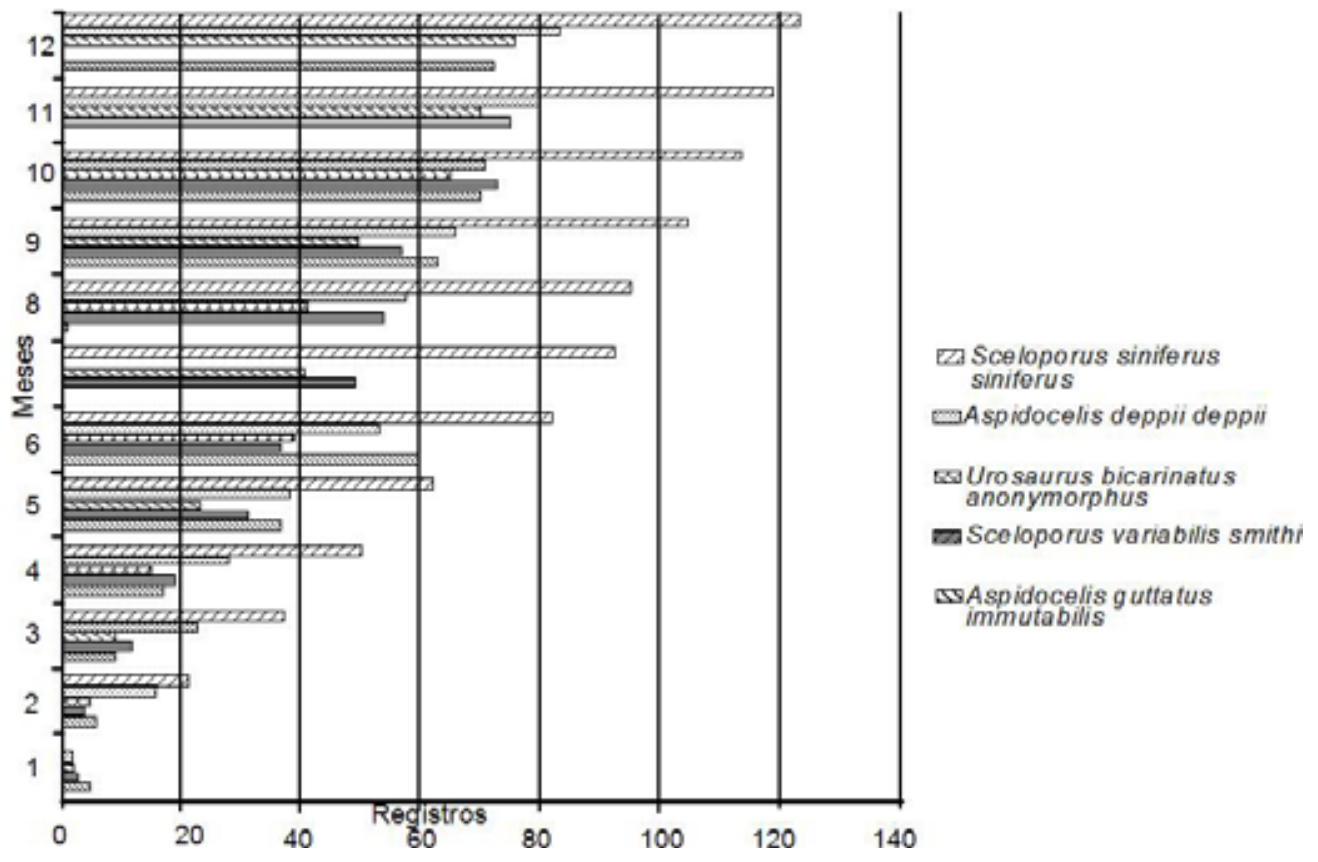

Figura 3. Abundancia de especies de herpetofauna del Cerro Guiengola, Tehuantepec, Oaxaca.

se registraron por encima del límite altitudinal superior reportado por estos autores y solamente C. oaxacana se observó por debajo del límite inferior (Cuadro 3).

Riqueza de especies por época del año. El número de especies observadas fue mayor en la época de lluvias (mayo-octubre) con 36 especies, mientras que en la época de secas (noviembre-abril) sólo se registraron 25 especies de anfibios y reptiles.

Curva de acumulación de especies. Las curvas de acumulación de especies mostraron un incremento creciente que no refleja una asíntota al término del año de muestreo, por lo que aún quedan especies de anfibios y reptiles por añadir al listado (Fig. 4). El cálculo de las especies esperadas con el estimador Chao2 da un máximo de 54 especies, lo que indica que se registró el $74.07 \%$ de la riqueza herpetofaunística calculada para el Cerro Guiengola.

De acuerdo con los Modelos de Clench y de Dependencia Lineal, para registrar el 95\% de las especies que se encuentran en el área de estudio se requieren efectuar 89 y 26 muestreos en los cuales se obtendrían 52 y 40 especies respectivamente. Lo anterior refleja que con el Modelo de Dependencia Lineal ya se registró el total de especies de anfibios y reptiles del Cerro Guiengola en un periodo inferior (12 muestreos) al que sugiere este modelo y para el Modelo de Clench es necesario continuar 
Cuadro 2. Distribución de los anfibios y reptiles en diferentes microhábitats y tipos de vegetación en el Cerro Guiengola, Tehuantepec, Oaxaca.

\begin{tabular}{|c|c|c|c|c|c|c|c|c|c|}
\hline Especies & \multicolumn{5}{|c|}{ Microhábitats } & \multicolumn{4}{|c|}{ Tipo de vegetación } \\
\hline & $\mathrm{R}$ & $\mathrm{T}$ & $\mathrm{HH}$ & $\mathrm{A}$ & $\mathrm{S}$ & VR & SBCE & SBC & $\mathrm{BP}$ \\
\hline Anolis compressicaudus & & $\mathrm{X}$ & & $\mathrm{X}$ & $\mathrm{X}$ & & $\mathrm{X}^{*}$ & $\mathrm{X}^{*}$ & \\
\hline Aspidoscelis deppii depii & $\mathrm{X}$ & $\mathrm{X}$ & & $X$ & $\mathrm{X}$ & $\mathrm{X}$ & $X^{*}$ & $\mathrm{X}^{*}$ & \\
\hline Aspidoscelis deppii oligoporus & $\mathrm{X}$ & $X$ & $\mathrm{X}$ & & & $\mathrm{X}$ & $\mathrm{X}^{*}$ & $\mathrm{X}^{*}$ & \\
\hline Aspidoscelis guttatus immutabilis & $X$ & $\mathrm{X}$ & & & $\mathrm{X}$ & $X^{*}$ & $\mathrm{X}^{*}$ & $\mathrm{X}^{*}$ & \\
\hline Basiliscus vittatus & $X$ & & & & & $\mathrm{X}$ & & & \\
\hline Boa constrictor & $\mathrm{X}$ & & & & & $\mathrm{X}^{*}$ & & & \\
\hline Conophis vittatus viduus & $\mathrm{X}$ & $\mathrm{X}$ & & & & $X^{*}$ & $X^{*}$ & & \\
\hline Crotalus durissus & & $\mathrm{X}$ & & & & & & $\mathrm{X}^{*}$ & \\
\hline Ctenosaura oaxacana & $\mathrm{X}$ & $\mathrm{X}$ & & $\mathrm{X}$ & $\mathrm{X}$ & $\mathrm{X}^{*}$ & $X^{*}$ & $\mathrm{X}^{*}$ & \\
\hline Ctenosaura pectinata & $\mathrm{X}$ & $\mathrm{X}$ & & $\mathrm{X}$ & $\mathrm{X}$ & $X^{*}$ & $\mathrm{X}$ & $\mathrm{X}^{*}$ & \\
\hline Drymarchon melanurus & & $\mathrm{X}$ & & & & & $X^{*}$ & & \\
\hline Eleutherodactylus pipilans & $X$ & & & & & $X^{*}$ & & & \\
\hline Hemidactylus frenatus & $\mathrm{X}$ & & $\mathrm{X}$ & $\mathrm{X}$ & & $\mathrm{X}^{*}$ & $X^{*}$ & $\mathrm{X}^{*}$ & \\
\hline Iguana iguana & $X$ & & & & & $X^{*}$ & & & \\
\hline Lepidophyma smithi & & & & & $\mathrm{X}$ & & & $\mathrm{X}^{*}$ & \\
\hline Leptodeira annulata & $X$ & & & & & $\mathrm{X}^{*}$ & & & \\
\hline Leptodeira nigrofasciata & & & $\mathrm{X}$ & & & & $X^{*}$ & & \\
\hline Leptotyphlops goudoti & & $\mathrm{X}$ & & & $\mathrm{X}$ & & & $\mathrm{X}^{*}$ & \\
\hline Loxocemus bicolor & & $\mathrm{X}$ & & & & & $X^{*}$ & & \\
\hline Mabuya unimarginata & $\mathrm{X}$ & $\mathrm{X}$ & & & & $X^{*}$ & & & $\mathrm{X}^{*}$ \\
\hline Masticophis mentovarius & & $\mathrm{X}$ & & & & & $\mathrm{X}^{*}$ & & \\
\hline Micrurus ephippifer & & $\mathrm{X}$ & & & & & & $\mathrm{X}$ & \\
\hline Ollotis canalifera & $X$ & $\mathrm{X}$ & & & & $\mathrm{X}^{*}$ & $X^{*}$ & & \\
\hline Oxybelis aeneus & & $\mathrm{X}$ & & & & & & $\mathrm{X}^{*}$ & \\
\hline Phrynosoma asio & $\mathrm{X}$ & $\mathrm{X}$ & & & & $X^{*}$ & $\mathrm{X}^{*}$ & & \\
\hline Phyllodactylus lanei & & & & $\mathrm{X}$ & $\mathrm{X}$ & & $X^{*}$ & $\mathrm{X}$ & \\
\hline Porthidium dunni & & $\mathrm{X}$ & $\mathrm{X}$ & $\mathrm{X}$ & $\mathrm{X}$ & & $X^{*}$ & $\mathrm{X}$ & \\
\hline Rhinella marina & $\mathrm{X}$ & $\mathrm{X}$ & $\mathrm{X}$ & & & $X^{*}$ & $X^{*}$ & & \\
\hline Rhinoclemmys rubida rubida & & $\mathrm{X}$ & & & & & $X^{*}$ & $\mathrm{X}^{*}$ & \\
\hline Salvadora lemniscata & & $\mathrm{X}$ & & $X$ & & & $X^{*}$ & $X^{*}$ & \\
\hline Sceloporus edwardtaylori & $\mathrm{X}$ & & $\mathrm{X}$ & $\mathrm{X}$ & $\mathrm{X}$ & $X^{*}$ & $X^{*}$ & $\mathrm{X}$ & \\
\hline Sceloporus melanorhinus melanorhinus & & & & $\mathrm{X}$ & $\mathrm{X}$ & & & $\mathrm{X}$ & \\
\hline Sceloporus siniferus siniferus & $\mathrm{X}$ & $\mathrm{X}$ & & $\mathrm{X}$ & $\mathrm{X}$ & $X^{*}$ & $X^{*}$ & $\mathrm{X}$ & \\
\hline Sceloporus variabilis smithi & & $\mathrm{X}$ & & $\mathrm{X}$ & $\mathrm{X}$ & & $X^{*}$ & $\mathrm{X}$ & \\
\hline Sphaerodactylus glaucus & & $X$ & $\mathrm{X}$ & $\mathrm{X}$ & $\mathrm{X}$ & & $X^{*}$ & $X^{*}$ & \\
\hline Symphimus leucostomus & & $X$ & & & & & & $\mathrm{X}^{*}$ & \\
\hline Tantilla rubra & & $\mathrm{X}$ & & & & & & $X^{*}$ & \\
\hline Trimorphodon biscutatus & $X$ & & $\mathrm{X}$ & & & $\mathrm{X}^{*}$ & $X^{*}$ & & \\
\hline Triprion spatulatus reticulatus & & & & $\mathrm{X}$ & & & $X^{*}$ & & \\
\hline Urosaurus bicarinatus anonymorphus & $\mathrm{X}$ & $\mathrm{X}$ & $\mathrm{X}$ & $\mathrm{X}$ & $\mathrm{X}$ & $\mathrm{X}^{*}$ & $X^{*}$ & $\mathrm{X}$ & \\
\hline Total & 20 & 27 & 9 & 15 & 15 & 20 & 26 & 24 & 1 \\
\hline
\end{tabular}

Ripario (R), Terrestre (T), Paredes y grietas de habitaciones humanas (HH), Arborícola (A) y Saxícola (S).

Vegetación riparia (VR), Selva baja caducifolia espinosa (SBCE) Selva baja caducifolia (SBC) y Bosque de pino (BP).

* Aportaciones del presente estudio. 
Martín-Regalado et al.: Herpetofauna del Cerro Guiengola, Oaxaca

Cuadro 3. Distribución altitudinal de los anfibios y reptiles en el Cerro Guiengola, Tehuantepec, Oaxaca.

\begin{tabular}{|c|c|c|}
\hline \multirow[t]{2}{*}{ Especies } & \multicolumn{2}{|c|}{ Altitud } \\
\hline & Este estudio & Casas-Andreu et al. (1996) \\
\hline Anolis compressicaudus & $120-917$ & $100-1000$ \\
\hline Aspidoscelis deppii depii & $45-765$ & $0-1800$ \\
\hline Aspidoscelis deppii oligoporus & $58-174$ & $0-1800$ \\
\hline Aspidoscelis guttatus immutabilis & $45-762$ & $0-1700$ \\
\hline Basiliscus vittatus & $45-93$ & $20-600$ \\
\hline Boa constrictor & 51 & $0-1500$ \\
\hline Conophis vittatus viduus & $87-108$ & $0-1000$ \\
\hline Crotalus durissus & 605 & $0-1000$ \\
\hline Ctenosaura oaxacana & $55-605^{*}$ & $100-300$ \\
\hline Ctenosaura pectinata & $48-639$ & $0-1000$ \\
\hline Drymarchon melanurus & $53-90$ & $0-1000$ \\
\hline Eleutherodactylus pipilans & 49 & $140-1600$ \\
\hline Hemidactylus frenatus & $45-781$ & $0-1000$ \\
\hline Iguana iguana & 55 & $0-700$ \\
\hline Lepidophyma smithi & 385 & $100-1400$ \\
\hline Leptodeira annulata & 92 & $100-2300$ \\
\hline Leptodeira nigrofasciata & 91 & $0-750$ \\
\hline Leptotyphlops goudoti & $345-709$ & $0-1400$ \\
\hline Loxocemus bicolor & 69 & $0-500$ \\
\hline Mabuya unimarginata & 74-998 & $0-1800$ \\
\hline Masticophis mentovarius & 110 & $0-1700$ \\
\hline Micrurus ephippifer & 624 & $200-2500$ \\
\hline Ollotis canalifera & $57-115$ & $0-200$ \\
\hline Oxybelis aeneus & 605 & $0-1500$ \\
\hline Phrynosoma asio & $83-103$ & $0-200$ \\
\hline Phyllodactylus lanei & $145-348$ & $0-1000$ \\
\hline Porthidium dunni & $91-650 *$ & $0-500$ \\
\hline Rhinella marina & $78-122$ & $0-1000$ \\
\hline Rhinoclemmys rubida rubida & $91-624$ & $5-1200$ \\
\hline Salvadora lemniscata & $59-778$ & $50-1200$ \\
\hline Sceloporus edwardtaylori & $43-564 *$ & $0-200$ \\
\hline Sceloporus melanorhinus melanorhinus & $282-473$ & $0-1600$ \\
\hline Sceloporus siniferus siniferus & $46-914$ & $0-2150$ \\
\hline Sceloporus variabilis smithi & $86-793$ & $0-1600$ \\
\hline Sphaerodactylus glaucus & $106-298^{*}$ & \\
\hline Symphimus leucostomus & 605 & $200-1000$ \\
\hline Tantilla rubra & 556 & $100-2200$ \\
\hline Trimorphodon biscutatus & $88-106$ & $0-1500$ \\
\hline Triprion spatulatus reticulatus & 91 & $50-200$ \\
\hline Urosaurus bicarinatus anonymorphus & $45-438$ & $0-1300$ \\
\hline
\end{tabular}

* Aportaciones del presente trabajo. 


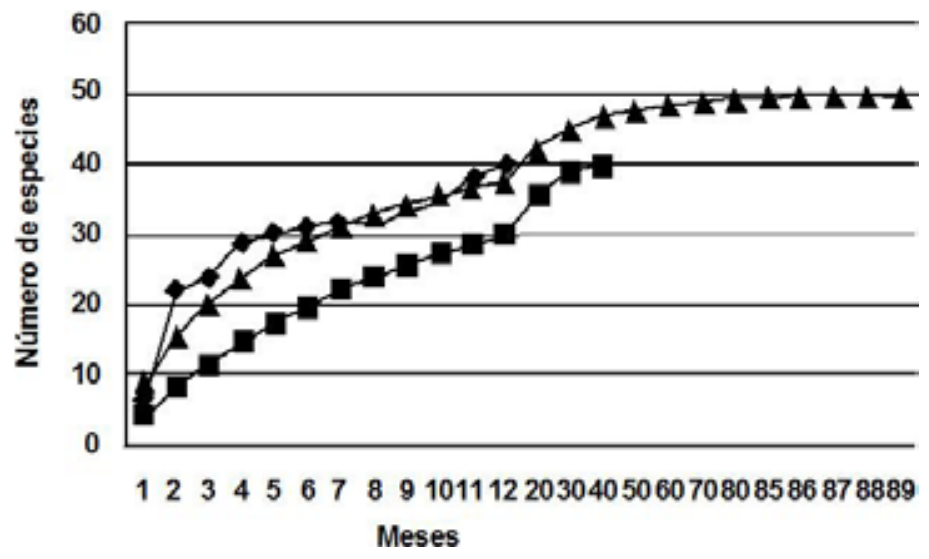

Figura 4. Número de especies de herpetofauna del Cerro Guiengola observadas ( $\bullet$ en un año de muestreo y estimadas con los modelos de Dependencia Lineal (a) y Clench $(\boldsymbol{\Delta})$.

con el muestreo, lo que se justifica si consideramos que faltan por añadir al listado aquellas especies raras o difíciles de observar.

Los pobladores de las comunidades cercanas al sitio de estudio mencionaron que en el área existen el escorpión y el cantil, cuyas descripciones probablemente corresponden a Heloderma horridum y Agkistrodon bilineatus que fueron reportadas por Casas-Andreu et al. $(1996,2004)$ para el Istmo de Tehuantepec. Sin embargo, dado que estas especies no fueron registradas durante este estudio no se pueden incluir como parte de la riqueza herpetofaunística del Cerro Guiengola.

Diversidad alfa. El índice de Simpson se acercó a 0 con valores de 0.08 en lluvias y 0.12 en secas, lo que refleja una mayor dominancia de especies durante la temporada de secas influenciada por las especies de los géneros Sceloporus y Aspidocelis.

El índice de Shannon-Wiener refleja una mayor equitatividad en lluvias (2.81) que en secas (2.46) debido a que la mayoría de las especies están representadas por igual número de registros (33) siendo pocas las especies dominantes (7) para ambas épocas del año. La prueba de Hutcheson reveló que la diversidad entre épocas es estadísticamente diferente.

Diversidad beta. En el análisis por microhábitat se observa que el arborícola y saxícola tienen un $66.88 \%$ de similitud al compartir 12 especies de anfibios y reptiles; el terrestre se agrupa con los anteriores con un $35.40 \%$ con ocho especies y el ripario con un $34.17 \%$ de similitud y siete especies compartidas. El microhábitat de paredes y grietas en habitaciones humanas fue el más disímil, uniéndose a los demás microhábitats, con un valor de $26.08 \%$ (Fig. 5). 


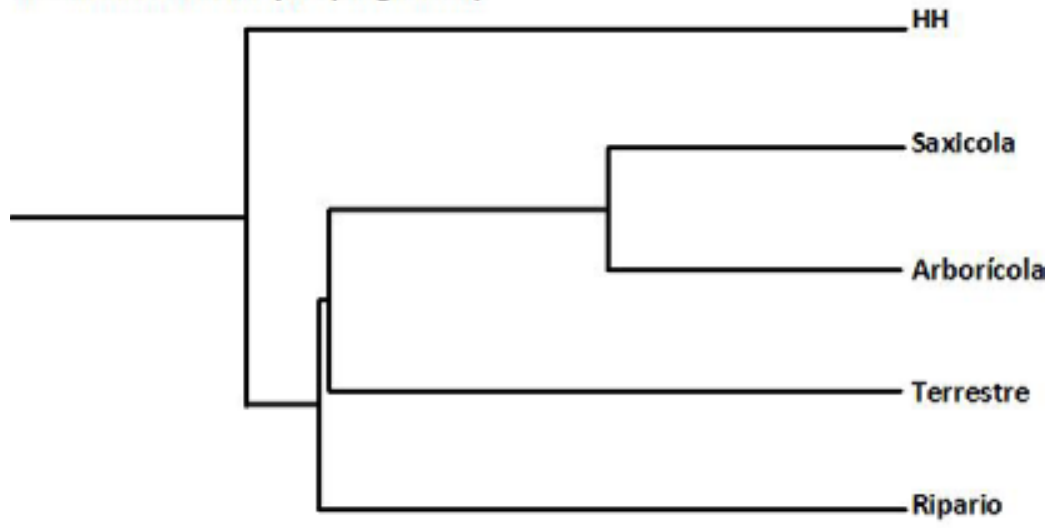

$\longdiv { 0 , \% \text { Similarity } 5 0 . } 1 0 0 .$

Figura 5. Dendrograma de similitud para cinco tipos de microhábitats en el Cerro Guiengola, Tehuantepec, Oaxaca. HH: Paredes y grietas de habitaciones humanas.

En el dendrograma de similitud para los diferentes tipos de vegetación, de acuerdo al método de Jaccard, se observa que la selva baja caducifolia y caducifolia espinosa son las más parecidas, debido a que comparten 16 especies y un valor de semejanza del $47.18 \%$, a estas se les une la vegetación riparia, con 14 especies compartidas y una similitud de $43.49 \%$; el bosque de pino es $4.97 \%$ similar a la vegetación riparia, compartiendo solo una especie (Mabuya unimarginata). El bosque de pino no comparte especies con las selvas bajas (Fig. 6).

Especies en categorías de riesgo. De las 40 especies que se enlistan, 17 están consideradas en alguna categoría de riesgo según la NOM-059-ECOL-2001 (SEMARNAT 2002), representando el $42.5 \%$ de las especies registradas en el Cerro Guiengola, de las cuales 13 son endémicas de México y tres de Oaxaca. La Unión Internacional para la Conservación de la Naturaleza (IUCN 2009) enlista en diferentes categorías a 19 (47.5\%) de las 40 especies encontradas en el Cerro Guiengola. La CITES enlista a Iguana iguana, Boa constrictor y Loxocemus bicolor en el apéndice II, donde se incluyen a especies que no se encuentran necesariamente en peligro de extinción, pero cuyo comercio debe controlarse a fin de evitar una utilización incompatible con su supervivencia. La IUCN coloca a Ctenosaura oaxacana en la categoría de peligro crítico y la NOM-059-ECOL-2001 como amenazada. La única tortuga registrada, Rhinoclemmys $r$. rubida, se encuentra en la categoría de casi amenazada según la IUCN y como sujeta a protección especial según la NOM-059-ECOL-2001. El cora- 


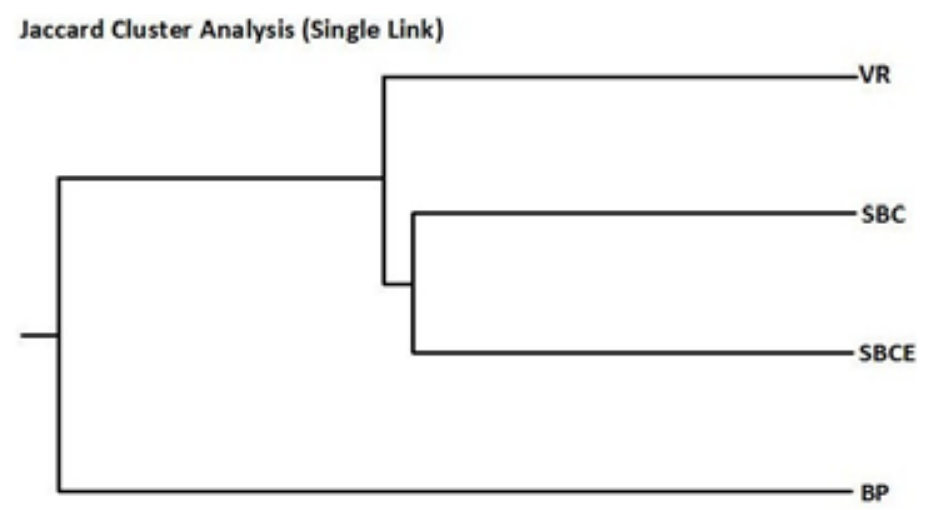

$\stackrel{0, \% \text { Similarity } \quad 50 .}{100 .}$

Figura 6. Dendrograma de similitud para los tipos de vegetación en el Cerro Guiengola, Tehuantepec, Oaxaca. VR: Vegetación riparia, SBC: Selva baja caducifolia, SBCE: Selva baja caducifolia espinosa, BP: Bosque de pino.

lillo endémico de Oaxaca (Micrurus ephippifer) se encuentra enlistada en la NOM059-ECOL-2001 en la categoría de sujeta a protección especial y en la IUCN como vulnerable (Cuadro 4).

\section{DISCUSIÓN}

En este estudio se registraron 40 especies, cuatro de anfibios y de 36 reptiles. Para la Región del Istmo de Tehuantepec, Casas-Andreu et al. (2004) reportaron 94 especies de reptiles (dos cocodrilos, 34 saurios, 51 serpientes, y siete tortugas) y 28 anfibios, de las cuales en el presente trabajo se registraron 16 serpientes $(28.07 \%), 19$ saurios (47.5\%), una tortuga (14.28\%) y cuatro anfibios (14.28\%). Lo anterior es resultado de la escasa presencia de cuerpos de agua y un sustrato rocoso que no permite la retención de humedad, lo que se ve reflejado en los tipos de vegetación dominantes en el Cerro Guiengola (selvas bajas caducifolias) favoreciendo la presencia de reptiles y restringiendo la presencia y distribución de anfibios.

Varias especies ocupan más de un microhábitat, y por lo general son aquéllas que no requieren un ambiente muy específico y presentan una amplia distribución; el caso más representativo es el de Urosaurus bicarinatus anonymorphus que se encontró en los cinco microhábitats, siendo en ese sentido una especie generalista. Las especies de los géneros Aspidoscelis y Sceloporus son de hábitos muy activos, desplazándose 
Cuadro 4. Especies en alguna categoría de riesgo según la NOM-059-ECOL-2001 y la IUCN 2009.

\begin{tabular}{|c|c|c|c|c|c|}
\hline Especies & Nombre Común & $\begin{array}{c}\text { Nom- } \\
059\end{array}$ & $\begin{array}{l}\text { End. } \\
\text { Oax }\end{array}$ & $\begin{array}{l}\text { End. } \\
\text { Mex }\end{array}$ & IUCN \\
\hline Anolis compressicaudus & & & & $*$ & $\mathrm{LC}$ \\
\hline $\begin{array}{l}\text { Aspidoscelis guttatus } \\
\text { immutabilis }\end{array}$ & & & & * & $\mathrm{LC}$ \\
\hline Boa constrictor & Boa constrictor-boa & A & & & \\
\hline Conophis vittatus viduus & & & & $*$ & $\mathrm{LC}$ \\
\hline Crotalus durissus & & $\operatorname{Pr}$ & & & \\
\hline Ctenosaura oaxacana & & A & * & & $\mathrm{CR}$ \\
\hline Ctenosaura pectinata & Iguana espinosa-mexicana & A & & $*$ & \\
\hline Eleutherodactylus pipilans & & & & & $\mathrm{LC}$ \\
\hline Iguana iguana & Iguana verde & $\operatorname{Pr}$ & & & \\
\hline Lepidophyma smithi & Lagartija nocturna de Smith & $\operatorname{Pr}$ & & & \\
\hline Leptodeira annulata & Culebra ojo de gato bandada & $\operatorname{Pr}$ & & & \\
\hline Loxocemus bicolor & Serpiente chatilla & $\operatorname{Pr}$ & & & \\
\hline Leptodeira nigrofasciata & & & & & $\mathrm{LC}$ \\
\hline Masticophis mentovarius & Culebra chirriadora neotropical & A & & * & \\
\hline Micrurus ephippifer & Serpiente coralillo oaxaqueña & $\operatorname{Pr}$ & * & & VU \\
\hline Ollotis canalifera & & & & & $\mathrm{LC}$ \\
\hline Phrynosoma asio & Lagartija-cornuda & $\operatorname{Pr}$ & & * & \\
\hline Porthidium dunni & Nauyaca nariz de cerdo oaxaqueña & $\mathrm{A}$ & & * & $\mathrm{LC}$ \\
\hline Rhinoclemmys $r$. rubida & Tortuga de monte payaso & $\operatorname{Pr}$ & & * & NT \\
\hline Rhinella marina & & & & & $\mathrm{LC}$ \\
\hline Salvadora lemniscata & Culebra parchada del Pacífico & $\operatorname{Pr}$ & & $*$ & $\mathrm{LC}$ \\
\hline Sceloporus edwardtaylori & & & $*$ & & $\mathrm{LC}$ \\
\hline Sceloporus m. melanorhinus & & & & * & $\mathrm{LC}$ \\
\hline Sceloporus s. siniferus & & & & & $\mathrm{LC}$ \\
\hline Sphaerodactylus glaucus & Geco enano collajero & $\operatorname{Pr}$ & & & \\
\hline Symphimus leucostomus & $\begin{array}{l}\text { Culebra labios blancos de } \\
\text { Tehuantepec }\end{array}$ & $\operatorname{Pr}$ & & $*$ & $\mathrm{LC}$ \\
\hline Trimorphodon biscutatus & Culebra-lira cabeza negra & $\operatorname{Pr}$ & & & $\mathrm{LC}$ \\
\hline Triprion spatulatus reticulatus & & & & $*$ & $\mathrm{LC}$ \\
\hline $\begin{array}{l}\text { Urosaurus bicarinatus } \\
\text { anonymorphus }\end{array}$ & & & & $*$ & $\mathrm{LC}$ \\
\hline
\end{tabular}

NOM-059-ECOL-2001: Sujeta a protección especial (Pr), Amenazada (A); IUCN: Preocupación menor (LC), Casi amenazado (NT), Vulnerable (VU) y En peligro crítico (CR). 
constantemente en busca de alimento en la selva baja caducifolia espinosa por lo que se les considera especies generalistas de hábitat. Las serpientes encontradas son predominantemente de hábitos terrestres, registrándose 12 especies en este ambiente, siendo tan solo cuatro las que se colectaron a orilla del río y dos en paredes y grietas en habitaciones humanas. Las especies que ocupan microhábitats muy específicos son Drymarchon melanurus y Leptotyphlops goudoti, las cuales son especialistas de hábitat, debido a que sólo se les observó sobre el suelo, al igual que a la única tortuga que se registró. Como grupo, los saurios son los que presentan mayor variedad de microhábitats explotados, seguidos de las serpientes.

Las especies más comunes en la selva baja caducifolia, selva baja caducifolia espinosa y vegetación riparia fueron Aspidoscelis d. deppii, Sceloporus variabilis smithi, S. siniferus siniferus y Urosaurus bicarinatus anonymorphus, estas dos últimas son especies bien conocidas por su tolerancia a la aridez (Canseco 1996), aunque su presencia en la vegetación riparia refleja que pueden tolerar ambientes más húmedos.

$\mathrm{Al}$ analizar la distribución altitudinal de las especies del Cerro Guiengola, se encontró que al aumentar la altitud, el número de especies decrece, no observándose un pico de riqueza específica a altitudes intermedias. Lo anterior puede estar determinado por la fisiología termal de los reptiles, temperatura del aire y del sustrato que influyen en la respuesta fisiológica o conductual de los organismos (Woolrich-Piña et al. 2006), limitando la distribución de especies como Phyllodactylus lanei a microhábitats específicos (cuevas con escasa iluminación y baja temperatura) con temperaturas inferiores a $\operatorname{los} 38^{\circ} \mathrm{C}$ registrados para el área de estudio en época de secas (Trejo 2004) y favoreciendo la amplia distribución de especies generalistas como Sceloporus s. siniferus y Anolis compressicaudus, las cuales dada su distribución parecen responder a las diferentes condiciones de los siete pisos altitudinales.

La presencia de las cuatro especies de anfibios registradas en el primer piso altitudinal durante el año de muestreo está relacionada con su cercanía a los cuerpos de agua y la época de lluvias, destacando la presencia de Triprion spatulatus reticulatus localizado en el hueco de un árbol cercano a un brote de agua esporádico, producto de la precipitación de ese año.

Al analizar la diversidad espacial del Cerro Guiengola se observa un mayor número de especies a altitudes inferiores que corresponden a las selvas bajas caducifolia (24 especies) y caducifolia espinosa (26 especies). La diversidad de especies por unidad de área es superior en la selva baja caducifolia espinosa ya que ésta ocupa una superficie menor con respecto al resto de tipos de vegetación presentes en el Cerro Guiengola, encontrándose de 0 a $350 \mathrm{~m} \mathrm{snm}$, con una mayor cercanía a las fuentes de agua (río las Tejas y río Tehuantepec), por lo que estos resultados pueden estar influenciados por los requerimientos de humedad de la herpetofauna. Dado que el bosque de pino se encuentra arriba de los $1000 \mathrm{~m}$ snm el número de especies registradas se encuentran influenciadas por la altitud y la vegetación. El patrón de diversidad 
observado en el sitio de estudio coincide con lo observado para especies terrestres y dulceacuícolas, que conforme se incrementa la altitud disminuye la riqueza de especies (Canseco 1996; Casas-Andreu 1996, 2004; Martínez-Ramírez 1999, 2004).

La protección de las especies reconocidas bajo alguna categoría de riesgo y endémicas de Oaxaca se considera prioritaria, particularmente para Ctenosaura oaxacana, Sceloporus edwardtaylori y Micrurus ephippifer. También es prioritaria la protección de 13 especies más que son endémicas de México y que están presentes en el Cerro Guiengola (Cuadro 4). Es importante tomar en cuenta el impacto del uso tradicional de la fauna por los habitantes de las Tejas, Santa María Mixtequilla y Santa María Jalapa del Márquez, quienes suelen consumir a C. oaxacana poniendo en riesgo su existencia en el área de estudio. Por lo anterior es recomendable establecer proyectos productivos de bajo impacto que mitiguen la presión antropogénica sobre los recursos herpetofaunísticos del Cerro Guiengola y que se continúen realizando investigaciones que generen la línea base para su protección y conservación.

La distribución de la herpetofauna en los tipos de vegetación presentes en el Cerro Guiengola (Cuadro 2), indica la necesidad de conservar todo el mosaico de hábitats, en vista que la selva baja caducifolia y la selva baja caducifolia espinosa tienen un número similar de especies en peligro. Si se reconoce que Oaxaca es el estado más rico en anfibios y reptiles, el Cerro Guiengola debe ser incluido dentro de las estrategias a nivel estatal para la conservación. El Istmo de Tehuantepec es la tercera provincia florística-faunística más diversa del estado, particularmente a altitudes entre 0 a 1000 $\mathrm{m}$ para diversidad y entre 1200 y $2600 \mathrm{~m}$ en lo referente a endemismo (Casas-Andreu et al. 1996).

AGRADECIMIENTOS. Este trabajo es parte de los resultados de la tesis para obtener el grado de Licenciatura en Biología que presentó la primera autora en el Instituto Tecnológico del Valle de Oaxaca. Queremos agradecer al Sr. Armando Calderón por el interés, su indispensable participación y valiosa aportación de sus conocimientos en el trabajo de campo; y a los pobladores de las Tejas por su hospitalidad. Agradecemos al Biól. Mario C. Lavariega por la elaboración de los mapas del área de estudio y al Dr. Emilio Martínez Ramírez por las sugerencias acertadas a este documento. Asimismo agradecemos sinceramente a dos revisores anónimos que hicieron valiosas observaciones a este manuscrito.

\section{LITERATURA CITADA}

Álvarez del Toro, M. 1982. Los reptiles de Chiapas. 3ra ed. Instituto de Historia Natural del Estado. Tuxtla Gutiérrez, Chiapas. México.

Canseco-Márquez, L. 1996. Estudio preliminar de la herpetofauna en la Cañada de Cuicatlán y Cerro Piedra Larga, Oaxaca. Tesis de Licenciatura. Benemérita Universidad Autónoma de Puebla. Puebla.

Casas Andreu, G. \& J. C. McCoy. 1979. Anfibios y reptiles de México. Ed. Limusa. México.

Casas-Andreu, G., F. R. Méndez de la Cruz \& J. L. Camarillo. 1996. Anfibios y Reptiles de Oaxaca. Lista, distribución y conservación. Acta Zoológica Mexicana (n.s.), 69: 1-35. 
Casas-Andreu, G., F. R. Méndez-de la Cruz \& X. Aguilar-Miguel. 2004. Anfibios y reptiles, pp. 375-390. In: A. J. García-Mendoza, M. J. Ordóñez \& M. Briones-Salas (Eds.). Biodiversidad de Oaxaca. Instituto de Biología, UNAM-Fondo Oaxaqueño para la Conservación de la NaturalezaWorld Wildlife Fund, México.

Centro de Investigación en Matemáticas A. C. 2003. Species Accumulation Functions. Version Beta.

CITES. 2009. Apéndices I, II Y III. Convention International trade and endangered species of wild fauna and flora y UNEP. 47 pp. http://www.CITES.org

Colwell, R. K. 2009. EstimateS: Statistical estimation of species richness and shared species from samples. Version 8. http://viceroy.eeb.uconn.edu/estimates

CONABIO. 1999. "Uso de suelo y vegetación modificado por CONABIO". Escala 1: 1,000,000. Comisión Nacional para el Conocimiento y Uso de la Biodiversidad. Ciudad de México, México.

CONABIO. 2006. División Política Estatal. Escala 1:250,000. Instituto Nacional de Estadística, Geografía e Informática y Comisión Nacional para el Conocimiento y Uso de la Biodiversidad. México.

Flores-Villela, O. 1993. Herpetofauna mexicana. Lista anotada de las especies de anfibios y reptiles de México, cambios taxonómicos recientes y nuevas especies. Special Publication No. 17. Carnegie Museum of Natural History, Pittsburgh.

Flores-Villela, O. \& F. P. Gerez. 1994. Biodiversidad y conservación en México: vertebrados, vegetación y uso del suelo. Comisión Nacional para el Conocimiento y Uso de la Biodiversidad y Universidad Nacional Autónoma de México. México. D. F.

Flores-Villela, O., F. Q. Mendoza \& G. P. González. 1995. Recopilación de claves para la determinación de anfibios y reptiles de México. Publicaciones Especiales del Museo de Zoología "Alfonso L. Herrera" No. 10. Universidad Autónoma de México.

Flores-Villela, O. \& L. Canseco-Márquez. 2004. Nuevas especies y cambios taxonómicos para la herpetofauna de México. Acta Zoológica Mexicana (n.s.), 20: 115-144.

Frost, D.R., T. Grant, J. N. Faivovich, R. H. Bain, A. Haas, C. F.B. Haddad, R.O. De Sá, A. Channing, M. Wilkinson, S. C. Donnellan, C. J. Raxworthy, J. A. Campbell, B. L. Blotto, P. Moler, R. C. Drewes, R. A. Nussbaum, J. D. Lynch, D. M. Green \& W. C. Wheeler. 2006. The amphibian tree of life. Bulletin of the American Museum of Natural History, 297: 1-370.

García, E. 1988. Modificaciones al Sistema de Clasificación Climática de Köppen (para adaptarlo a las condiciones de la República Mexicana). 4a . ed. Offset Larios, México.

Gutiérrez-Mayén, M. G. \& J. A. Salazar. 2006. Herpetofauna de los municipios de Camocuautla, Zapotitlán de Méndez y Huitzilan de Serdán, de la Sierra Norte de Puebla, pp. 197-223. In: Ramírez-Bautista A., L. Canseco-Márquez \& F. Mendoza-Quijano Eds.). Inventarios herpetofaunísticos de México: avances en el conocimiento de su biodiversidad. Sociedad Herpetológica Mexicana, A. C. México, D. F.

Hartweg, N. \& J. A. Oliver. 1937a. A contribution to the herpetology of the Isthmus of Tehuantepec. I. Occasional Papers of the Museum of Zoology No. 356. University of Michigan Press.

Hartweg, N. \& J. A. Oliver. 1937b. A contribution to the herpetology of the Isthmus of Tehuantepec. II. Occasional papers of the Museum of Zoology No. 359. University of Michigan Press.

Hartweg, N. \& J. A. Oliver. 1938. A contribution to the herpetology of the Isthmus of Tehuantepec. III. Occasional papers of the Museum of Zoology No. 390. University of Michigan Press.

Hartweg N. \& J. A. Oliver. 1940. A contribution to the herpetology of the Isthmus of Tehuantepec. IV. Miscellaneous Publications. Museum of Zoology No. 47. University of Michigan.

IUCN. 2009. Categorías y Criterios de la Lista Roja de la IUCN. Versión 3.1. Comisión de Supervivencia de Especies de la IUCN. IUCN, Gland, Suiza y Cambridge, Reino Unido. 
Jiménez-Valverde \& A. J. Hortal. 2000. Las curvas de acumulación de especies y la necesidad de evaluar la calidad de los inventarios biológicos. Revista Ibérica de Aracnología, 8: 151-161.

Liner, A. E. 2007. A. Checklist of the amphibians and reptiles of México. Occasional Papers of the Museum of Natural Science Louisiana State University No. 80.

López, R. E. 1979. Geología de México. Tomo III. 2a ed. D. F., México.

Magurran, A. E. 1988. Ecological diversity and its measurement. Princeton University Press, Princeton, New Jersey.

Martínez-Ramírez, E. 1999. Taxonomía y zoogeografía de la ictiofauna dulceacuícola del estado de Oaxaca, México. Tesis doctoral. Departamento de Ecología. Universidad de Barcelona.

Martínez-Ramírez, E., I. Doadrio Villarejo \& A. de Sostoa Fernández. 2004. Peces continentales, pp. 357-373. In: A. J. García-Mendoza, M. J. Ordóñez \& M. Briones-Salas (Eds.). Biodiversidad de Oaxaca. Instituto de Biología, UNAM-Fondo Oaxaqueño para la Conservación de la NaturalezaWorld Wildlife Fund, México.

McAleence, N. 1997. BioDiversity Professional Beta. Version 2. The Natural History Museum \& The Scottish Association for Marine Sciencie.

Moreno, C. E. \& G. Halffter. 2000. Assessing the completeness of bat biodiversity inventories using species accumulation curves. Journal of Applied Ecology, 37: 149-158.

Moreno, C. E. 2001. Métodos para medir la biodiversidad. M\&T - Manuales y Tesis SEA, vol. 1. Zaragoza, España.

SEMARNAT. 2002. Norma Oficial Mexicana NOM-059-ECOL-2001. Protección ambiental-especies nativas de México de flora y fauna silvestres-Categorías de riesgo y especificaciones para su inclusión, exclusión o cambio-Lista de especies en riesgo. Diario Oficial de la Federación, 6 de marzo de 2002.

Smith, H. M. \& E. H. Taylor. 1945. An annotated checklist and key of the snakes of Mexico. United States National Museum Bulletin, 187: 1-239.

Smith, H. M. \& E. H. Taylor. 1948. An annotated checklist and key to the amphibia of Mexico. United States National Museum Bulletin, 194: 1-118.

Smith, H. M. \& E. H. Taylor. 1950. An annotated checklist and key to the reptiles of Mexico exclusive of the snakes. United States National Museum Bulletin, 199: 1-254.

Smith, H. M. \& E. H. Taylor. 1966. Herpetology of Mexico annotated checklist and key to the reptiles of Mexico. A reprint of United States National Museum Bulletin, 187, 194, 199 with a list of subsequent Taxonomic Innovations. Eric Lundberg, Ashton, Maryland.

Soberón, J. \& J. Llorente. 1993. The use of species accumulation functions for the prediction of species richness. Conservation Biology, 7: 480-488.

Torres-Colín, M. L. 1989. Estudio florístico y descripción de la vegetación del Cerro Guiengola, en el Istmo de Tehuantepec, Oaxaca. Tesis de Licenciatura, ENEP-Iztacala, UNAM, México.

Torres-Colín, R. 2004. Tipos de vegetación, pp. 105-117. In: A. J. García-Mendoza, M. J. Ordóñez \& M. Briones-Salas (Eds.). Biodiversidad de Oaxaca. Instituto de Biología, UNAM-Fondo Oaxaqueño para la Conservación de la Naturaleza-World Wildlife Fund, México.

Trejo, I. 2004. Clima, pp. 67-85. In: A. J. García-Mendoza, M. J. Ordóñez \& M. Briones-Salas (Eds.). Biodiversidad de Oaxaca. Instituto de Biología, UNAM-Fondo Oaxaqueño para la Conservación de la Naturaleza-World Wildlife Fund, México.

Whittaker, R. H. 1972. Evolution and measurement of species diversity. Taxon, 21: 213-251.

Woolrich-Piña, G. A., J. A. Lemos-Espinal, L. Oliver-López, M. E. Calderón-Méndez, J. E. González-Espinoza, F. Correa-Sánchez \& R. Montoya Ayala. 2006. Ecología térmica de una población de la lagartija Sceloporus grammicus (Iguanidae: Phrynosomatinae) que ocurre en la zona Centro-Oriente de la Ciudad de México. Acta Zoológica Mexicana (n.s.), 22: 137-150. 